\title{
Low Back Pain During Streptokinase Infusion
}

\author{
Ronald Feitosa Pinheiro, Adriana Sanches Pedrolo, Marcelo Nigri, Antonio Carlos de Camargo Carvalho \\ São Paulo, SP - Brazil
}

We report the case of a 57-year-old male patient with severe low back pain during streptokinase infusion administered to treat typical chest pain and elevation of the ST segment in the inferior wall. We reviewed the literature, emphasizing the differential diagnosis, the pathophysiology, and management of the event.

The treatment of acute myocardial infarction in its first hours of evolution is currently based on reperfusion of the culprit artery, as soon as possible, with a fibrinolytic agent or primary angioplasty. Streptokinase is one of the fibrinolytic agents currently used. It is a highly active thrombolytic substance produced by strains of the group C beta-hemolytic streptococci according to the Lancefield classification. Of the most common adverse effects resulting from the use of streptokinase, we may cite hypotension, bleeding at the sites of puncture, urticaria, redness, nausea, vomiting, and headache ${ }^{1}$. Hemorrhagic stroke is one of the most severe side effects, and it occurs in $0.3 \%$ to $1 \%$ of the patients using that thrombolytic agent.

We report another side effect occurring during streptokinase infusion, low back pain, which has already been cited in some case reports since 1990.

\section{Case Report}

The patient is a 57-year-old male with diabetes and chronic renal insufficiency, who was admitted to the Coronary Unit of the Hospital São Paulo of the Escola Paulista de Medicina of the Universidade Federal de São Paulo (UNIFESP) complaining of typical chest pain for 4 hours. His electrocardiogram showed elevation of the ST segment in the inferior wall. On physical examination, the patient was hemodynamically stable, his cardiac and pulmonary auscultations were normal, and his peripheral pulses were palpable and symmetric.

Infusion of the thrombolytic agent was initiated, and,

Escola Paulista de Medicina - UNIFESP

Mailing address: Ronald Feitosa Pinheiro - Rua Napoleão de Barros, 715 10ªndar - 04024-002 - São Paulo, SP, Brazil - E-mail: carvalho.dmed@epm.br English version by Stela Maris C. e Gandour after receiving 130,000 $\mathrm{U}$, the patient began to complain of severe bilateral low back pain, which he characterized as excruciating and radiating to the hypogastric region. The thrombolytic infusion was suspended for 5 minutes. The physical examination of the patient at that time remained unaltered with maintenance of the blood pressure in his 4 limbs, palpable and symmetric peripheral pulses, and normal neurological examination. Based on the normal physical examination and considering the benefits of continuing the thrombolytic agent, we decided to take the chance that the back pain would not recur and maintained the thrombolytic infusion. When restarting the drug, the patient complained again of low back pain. We initiated analgesia with $5 \mathrm{mg}$ of a decimal solution of meperidine, maintained thrombolytic infusion at a lower pace, and from the $12^{\text {th }}$ minute of infusion on, the patient began to report significant relief in low back pain, and no longer complained of chest pain. During the rest of the infusion, the patient still complained of low back pain, which was mild and bearable. Ten minutes after the end of infusion, the patient no longer complained of any pain.

On the same day, the patient underwent abdominal echography, which revealed signs of chronic parenchymal nephropathy, but no signs of aortic dissection or retroperitoneal hemorrhage.

\section{Discussion}

In 1990, Shah and Taylor ${ }^{2}$, for the first time, reported 3 cases of low back pain during streptokinase infusion. The first patient was a 54-year-old hypertensive male, who was admitted to the hospital with severe chest pain and electrocardiographic findings of infarction in the anterior wall. The patient received acetylsalicylic acid, and streptokinase was started. After infusion of $350,000 \mathrm{U}$, the patient reported severe central low back pain; therefore, the thrombolytic infusion was suspended. Five minutes after drug suspension, the patient no longer complained of low back pain.

The $2^{\text {nd }}$ patient was a 35-year-old diabetic male complaining of chest pain for the previous 2 hours. His electrocardiogram showed anterolateral infarction. After receiving $300,000 \mathrm{U}$ of streptokinase, the patient complained of severe 
low back pain with irradiation to the lower limbs. The thrombolytic agent was suspended, and the pain was relieved in 5 minutes.

The $3^{\text {rd }}$ patient was a 64-year-old male with antecedent stable angina, who reported excruciating low back pain after receiving 255,000 $\mathrm{U}$ of streptokinase, the drug being then suspended. The patient was medicated with hydrocortisone, which significantly improved his low back pain. The thrombolytic agent was restarted after the physical examination proved unchanged.

In this $1^{\text {st }}$ report, the authors only reintroduced the thrombolytic agent with a corticoid in the $3^{\text {rd }}$ patient, because they considered the event to be an allergic manifestation to the drug. Shah et $\mathrm{al}^{2}$ did not report whether the patients were further investigated.

In 1991, Dickinson and Rosser ${ }^{3}$ reported the cases of 2 patients with low back pain during streptokinase infusion. Their $1^{\text {st }}$ patient was a 74-year-old hypertensive male with chest pain and an electrocardiogram compatible with infarction in the inferior wall. After administration of $150,000 \mathrm{U}$ of streptokinase, the patient experienced severe low back pain, which subsided after intravenous administration of $5 \mathrm{mg}$ of morphine and suspension of the thrombolytic agent. Their $2^{\text {nd }}$ patient was a 73 -year-old diabetic female with chest pain, dyspnea, and an electrocardiogram compatible with infarction in the lateral wall. After infusion of $200,000 \mathrm{U}$ of streptokinase, the patient complained of severe low back pain, the thrombolytic agent being then suspended. The pain spontaneously disappeared within 10 minutes. The patient evolved with the appearance of a rash on her lower limbs, which led the authors to confirm the pathophysiology suggested by Shah and Taylor that the phenomenon could be an allergic manifestation.

In the same year, Porter and Nikoletatos ${ }^{4}$ reported the case of a 50-year-old female with typical chest pain and an electrocardiogram compatible with infarction in the inferior wall, who, after starting streptokinase infusion, began to complain of symptoms similar to those already cited. The thrombolytic agent was suspended.

In October 1992, Lear et al ${ }^{5}$ reported on 5 patients with low back pain during streptokinase infusion. In 3 of these patients, the pain was eliminated with $2.5 \mathrm{mg}$ of intravenous morphine, streptokinase being maintained. No adverse effect was observed in those who finished infusion. Their $4^{\text {th }}$ patient complained of the reappearance of the pain after reintroduction of the drug, whose infusion was then suspended. In the $5^{\text {th }}$ patient, streptokinase was replaced by tissue plasminogen activator, and the patient no longer complained of pain. The authors emphasized that no patient developed a rash, hypotension, urticaria, edema glottidis, or any other symptom suggesting an allergic manifestation. As all 5 patients had enzyme findings compatible with acute myocardial infarction, the authors raised the hypothesis that the low back pain could have resulted from an effect secondary to lysis of an intracoronary thrombus.

In the same year, new reports on this effect of streptokinase appeared, but with different clinical characteristics. Ma- hadeva and Siklos ${ }^{6}$ reported 2 cases. The $1^{\text {st }}$ patient had an electrocardiogram compatible with infarction in the lateral wall, and, after 30 minutes of streptokinase infusion, he complained of severe interscapular pain. The thrombolytic agent was suspended, and the pain subsided spontaneously within 5 minutes. The $2^{\text {nd }}$ patient was a 60 -year-old female with clinical and electrocardiographic findings compatible with infarction, who, after 15 minutes of streptokinase infusion, complained of severe pain in the inferior region of the hypogastrium. In that report ${ }^{6}$, the authors suggested that streptokinase could not only cause low back pain, but also abdominal pain.

Still in 1992, a new report showed an evolution different from that reported until that time. Jenkins ${ }^{7}$ reported the case of a patient with low back pain during streptokinase infusion, who had gross hematuria at the beginning of infusion and evolved to microscopic hematuria in the 12 subsequent hours. The thrombolytic agent was suspended and imaging examinations showed no abnormalities.

In November 1992, Bourke ${ }^{8}$ reported 3 new cases of low back pain during streptokinase infusion. In the 3 cases, the pain was extremely severe and had the characteristics already cited. In all of them, streptokinase was suspended, and an endovenous opioid was administered, which resulted in relief of the low back pain in less then 10 minutes.

In 1995, Fishwick et al ${ }^{9}$ reported on the case of a 66year-old patient with chest pain for 6 hours and an electrocardiogram indicating infarction in the posterior wall ${ }^{9}$. After infusion of 250,000 U of streptokinase, the patient suddenly developed severe low back pain. As no neurologic abnormality or vascular alteration could be detected on physical examination, the authors replaced streptokinase with a tissue plasminogen activator. The treatment was completed with no other incidents, and no further investigation was reported.

On a review of the literature, we found no new cases reported in the last 5 years. Therefore, we conclude that since the initial report of 3 cases by Shah and Taylor ${ }^{2}, 16$ new cases have been reported. In only 4 patients, streptokinase was maintained after a brief clinical assessment and use of opioids. In 2 patients, streptokinase was replaced by tissue plasminogen activator, and the infusion could be maintained. In the remaining patients, the thrombolytic agent was suspended, and relief of the low back pain always occurred in less than 10 minutes, independent of opioid use. This may serve to differentiate low back pain from aortic dissection, because the latter does not usually subside spontaneously and may be refractory to opioids.

The 2 major differential diagnoses of this event are aortic dissection and retroperitoneal hemorrhage. The imaging techniques are extremely important for this differentiation, which cannot be made through a simple physical examination. In an ideal situation, an imaging examination should be performed prior to the safe reintroduction of the thrombolytic agent. In the cases in which streptokinase was reintroduced, no imaging examination was performed. In our patient, the physical examination remained unaltered, and the low back pain practically subsided after drug suspension. As we were not able to perform an imaging exami- 
nation, we assessed the risk-benefit ratio and reintroduced the thrombolytic agent.

In conclusion, based on a literature review, we found reports of 19 cases of low back pain during streptokinase infusion. No patient died due to this complication. The incidence of this phenomenon is difficult to establish in a reliable manner due to the small number of cases reported.

Further studies are definitely necessary to thoroughly assess this phenomenon. What is its pathophysiology? The theories proposed have not been scientifically evaluated. The entire literature on this subject has been based on case reports, which makes a more reliable assessment extremely difficult. Therefore, low back pain during streptokinase infusion is clearly an open question, which requires deeper studies to better understand this very interesting phenomenon associated with streptokinase infusion.

\section{References}

1. Goodman \& Gilman. The Pharmacological Basis of the Therapeutics. McgrawHill, $9^{\text {th }}$ edition, 1996: 1353 .

2. Shah M, Taylor RT. Low back pain associated with streptokinase. BMJ 1990; 301: 1219.

3. Dickinson RJ, Rosser A. Low back pain associated with streptokinase. BMJ 1991; 302: 111-2.

4. Porter NJ, Nikoletatos K. Low back pain associated with streptokinase. BMJ 1991; 302: 111-2.
5. Lear J, Rajapakase R, Pohl J. Low back pain associated with streptokinase. Lancet 1992; 340: 851.

6. Mahadeva R, Siklos PW. Pain during streptokinase infusion. Lancet 1992; 340: 1234-5.

7. Jenkins RD. Pain during streptokinase infusion. Lancet 1992; 340: 1235

8. Bourke J. Streptokinase and low back pain. NZ Med J 1992; 105: 482.

9. Fishwick D, Prasan A, Adams P. Thrombolysis and low back pain. BMJ 1995; 310; 504. 